\title{
The Multicriteria Constrained Stochastic Matched Filter For Underwater Bioacoustic Signals
}

\author{
Bernard Xerri \\ IM2NP \\ Aix Marseille Univ., Univ. Toulon, CNRS, \\ Marseille, France \\ bernard.xerri@univ-tln.fr
}

\author{
Bruno Borloz \\ IM2NP \\ Aix Marseille Univ., Univ. Toulon, CNRS, \\ Marseille, France \\ bruno.borloz@univ-tln.fr
}

\author{
Maissa Chagmani \\ IM2NP \\ Aix Marseille Univ., Univ. Toulon, CNRS, \\ Marseille, France \\ maissa.chagmani@univ-tln.fr
}

\begin{abstract}
The aim of this paper is the detection of a bioacoustic signal embedded in several noises such as sea noise and other bioacoustic signals (dolphins, sperm whales). All the signals are real world signals.
\end{abstract}

Only second order statistics are use through the estimated correlation matrices of the signals.

This paper proposes an extension of the Constrained Stochastic Matched Filter (CSMF) based on the optimization of the Signal to Noise Ratio after linear filtering. The approach proposed is a multicriteria one, merging three different versions of the CSMF, and is named Multicriteria CSMF (MCSMF).

The objective is that the results obtained are better than the other methods, or at least equal to the best among the three.

The results are provided on ROC curves and the method is compared to the classical method Stochastic Matched Filter (SMF).

Keywords-detection, bioacoustics, Signal to Noise Ratio (SNR), stochastic matched filter, multi-noise, correlation matrix

\section{INTRODUCTION}

Detection of bioacoustic signals is an important area of research to, for instance, estimate animal density or abundance from acoustic data.

In this paper we consider dolphin and sperm whales signals embedded in sea noise : these signals are real world ones found in the SABIOD/Bombyx Database [1].

Dolphins can produce signals such as whistles or clicks. We are interested only in clicks (more difficult to detect). Our objective is to detect dolphins signals, without confusion with the others, with a linear filter which is an extension of the Stochastic Matched Filter (SMF), named Multicriteria Constrained Stochastic Matched Filter (MCSMF).

A test signal is created with realizations of dolphins and sperm whales clicks with a normalized energy, and sea noise the power of which can be modified to impose the signal to noise ratio (SNRsea). The sea noise is always there and the clicks are added up.

The method we propose is compared to two other classical method: the first one is the SMF for which the noise is only the sea noise. The second one is the SMF for which the noise is the mean of the undesired signals (sea noise and sperm whale signals).

All the methods tested only use correlation matrices of the different signals.

\section{The MUlticriteria CONSTRAined StOCHASTIC MATCHED FILTER}

We consider that the $n \times n$-dimension correlation matrices of each class of signals are known or estimated from data learning. Here we try to detect dolphin clicks (correlation matrix $\boldsymbol{A}$ ) when other signals are unwanted $\left(\boldsymbol{B}_{\mathbf{1}}, \boldsymbol{B}_{\mathbf{2}}\right.$ are the correlation matrices for sea noise and sperm whales).

\section{A. The SMF}

If the signal $s$ to detect were determinist, the optimal filter to use is the Matched Filter (MF) which consists in projecting the measured data onto a one dimensional $(p=1)$ subspace spanned by $\boldsymbol{B}^{-\mathbf{1}} \boldsymbol{S}$ ( $\boldsymbol{B}$ is the noise correlation matrix).

When the signal is stochastic, the question is: "is $p$ still equal to 1 for optimal detection ?".

Classically the Stochastic Matched Filter (SMF) is used when there is a useful signal, of correlation matrix $\boldsymbol{A}$, to detect and only one disturbing noise of correlation matrix $\boldsymbol{B}$.

It consists in calculating the eigenelements of $\boldsymbol{B}^{\mathbf{- 1}} \boldsymbol{A}$, projecting the measured data onto each eigenvectors and summing $p \geq 1$ powers of these terms. [2,3,4].

The optimal value of $p$ is chosen according to the different ROC curves. Nevertheless, the obtained $p$ dimensional subspace has no reason to be the one where the SNR is maximal. 
In a multi noise context, people generally consider a mean noise of correlation matrix $\boldsymbol{B}=\boldsymbol{B}_{\mathbf{1}}+\boldsymbol{B}_{\mathbf{2}}$ (which are the correlation matrices of the two undesired noises) and then use the SMF to solve this problem. It will be called SMF $\Sigma$ in the following.

\section{B. The CSMF}

Another approach consists in searching a $p$-dimension projection subspace $(p<n)$ spanned by a $n \times p$ orthonormal matrix $\boldsymbol{X}$, in which the Signal to Noise Ratio (SNR) given by $\rho=\frac{\operatorname{tr}\left(\boldsymbol{X}^{T} \boldsymbol{A} \boldsymbol{X}\right)}{\operatorname{tr}\left(\boldsymbol{X}^{T} \boldsymbol{B} \boldsymbol{X}\right)}$ is maximal.

Again, the optimal value of $p$ is chosen according to the different ROC curves, and has no reason to be the same than the SMF's one.

This filter is the Constrained Stochastic Matched Filter (CSMF) [5].

This has been extended in a multi noise context [6]. Then we can define for each noise a SNR $\rho_{\mathrm{i}}=\frac{\operatorname{tr}\left(\mathrm{X}^{\mathrm{T}} \mathrm{AX}\right)}{\operatorname{tr}\left(\mathrm{X}^{\mathrm{T}} \mathrm{B}_{\mathrm{i}} \mathrm{X}\right)}$. The objective is to maximize a criterium like $J=\sqrt{\prod_{i=1}^{2} \rho_{i}}$ or $\frac{1}{2} \sum_{i=1}^{2} \rho_{i}$ or $\frac{2}{\sum_{i=1}^{2} \frac{1}{\rho_{i}}}$ (geometric, arithmetic or harmonic mean).

All the correlation matrices are full-rank and normalized (trace equal to 1).

Those three criteria lead to a similar equation in which appear $\boldsymbol{A}$ and a weighted mean of the $\boldsymbol{B}_{\boldsymbol{i}}, \sum_{\mathrm{i}=1}^{2} \alpha_{\mathrm{i}} \mathrm{B}_{\mathrm{i}}$ (the $\alpha_{\mathrm{i}}$ depend of the chosen criterium). These equations are solved with the same algorithm (CSMF) which leads to $\boldsymbol{X}$ and the $\rho_{i}$ (hence $J$ ).

Denoting $\boldsymbol{X}_{p 1}, \boldsymbol{X}_{p 2}$ and $\boldsymbol{X}_{p 3} \in M_{n \times p}$ the $n \times p$-matrices of the vectors spanning the three optimal subspaces (geometric, arithmetic and harmonic), we calculate the power of the measured signal $x$ after projection onto $\boldsymbol{X}_{p i}, i=1,2,3$ :

$$
\operatorname{tr}\left(\mathrm{X}_{\mathrm{pi}}^{\mathrm{T}} \mathrm{xx}^{\mathrm{T}} \mathrm{X}_{\mathrm{pi}}\right)=\mathrm{x}^{\mathrm{T}} \mathrm{X}_{\mathrm{pi}} \mathrm{X}_{\mathrm{pi}}^{\mathrm{T}} \mathrm{X}
$$

which is used to perform detection (with a threshold).

Note that the harmonic criterium leads to the CSMF solution using $\boldsymbol{B}=\boldsymbol{B}_{1}+\boldsymbol{B}_{2}$.

\section{The MCSMF}

The experiments show that no criterium is always better than the others. Hence, a new idea consists in merging the three optimal subspaces after a power normalization. This is the Multicriteria CSMF.

We define

$$
\boldsymbol{X}_{p m}=\left[\frac{\boldsymbol{X}_{p 1}}{\sqrt{\beta_{1}}} \frac{\boldsymbol{X}_{p 2}}{\sqrt{\beta_{2}}} \frac{\boldsymbol{X}_{p 3}}{\sqrt{\beta_{3}}}\right] \in M_{n \times(3 p)}
$$

with $\beta_{i}=\operatorname{tr}\left(\boldsymbol{X}_{p i}^{\mathrm{T}} \boldsymbol{A} \boldsymbol{X}_{p i}\right), i=1,2,3$. Then we calculate the power of the measured signal $\boldsymbol{x}$ after projection onto $\boldsymbol{X}_{p m}$ : $\operatorname{tr}\left(\boldsymbol{X}_{p m}^{\mathrm{T}} \boldsymbol{x} \boldsymbol{x}^{T} \boldsymbol{X}_{p m}\right)=\boldsymbol{x}^{T} \boldsymbol{X}_{p m} \boldsymbol{X}_{p m}^{\mathrm{T}} \boldsymbol{x}$ which is used to perform detection (with a threshold).
The hope is that the results obtained are better than the other methods, or at least equal to the best among the three.

\section{EXPERIMENTS}

From the SABIOD database, we have extracted a few realizations of bioacoustics signal (dolphins and sperm whales). It is a hard and time-consuming task because the database is poorly informed: hence we have only 15 realizations of clicks for each category.

However, the sea noise is easy to extract and we have a long record available.

\section{A. The bioacoustic signals}

Fig. 1 shows one out of the 15 realizations of dolphin clicks: its length is 150 samples $\left(f_{s}=44 \mathrm{kHz}\right)$.

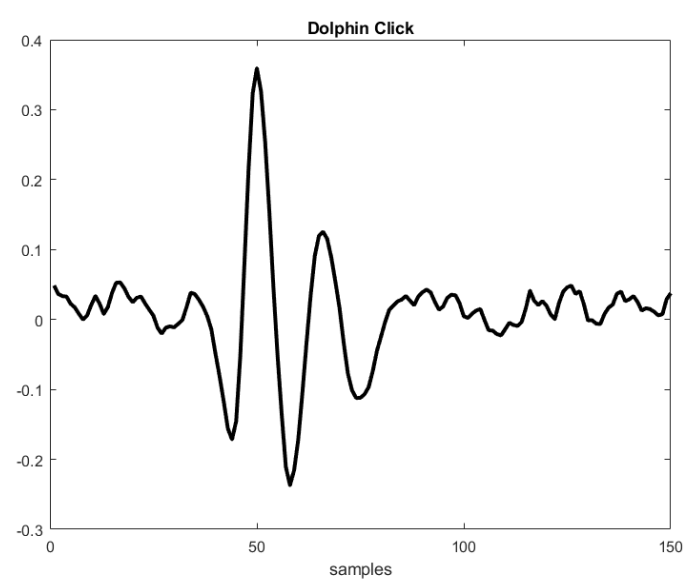

Fig. 1. Dolphin click

Fig. 2 shows one out of the 15 realizations of sperm whales clicks: its length is 150 samples $(f s=44 \mathrm{kHz})$.

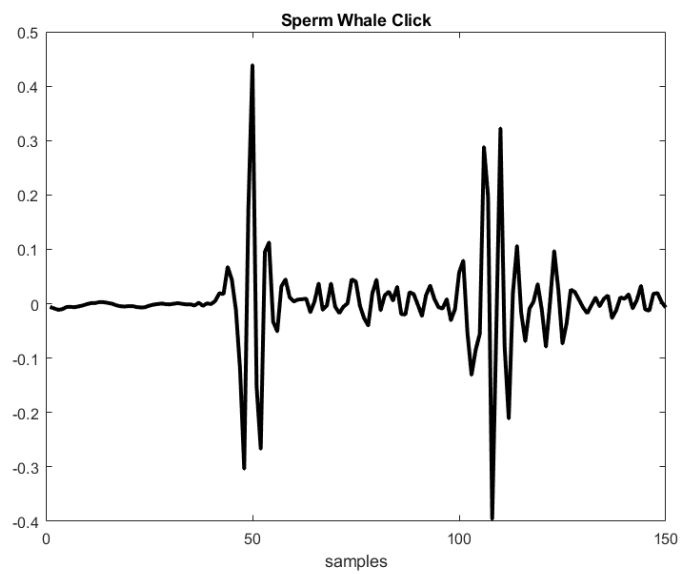

Fig. 2. Sperm Whale click 


\section{B. The test signal}

A test signal is created by addition of different realizations of the bioacoustic signals in sea noise which is present everywhere.

The test signal contains 40 realizations of dolphins clicks and 40 realizations of sperm whales clicks. Necessarily there are repetitions of some realizations, but however the sea noise is each time different and so the situation is never the same.

We don't have the choice, having a few number of realizations and must use some of them several times: this is not a satisfying situation but it is necessary to estimate acceptable ROC curves.

Fig. 3 shows the test signal for a SNR (vs. sea noise) of $6 \mathrm{~dB}$ : dolphins clicks are present only in the tagged areas.

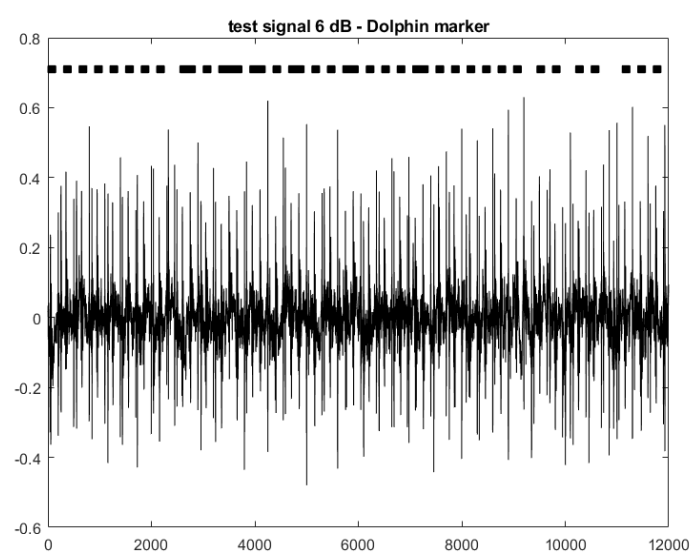

Fig. 3. Test Signal $($ SNRsea $=6 \mathrm{~dB})$

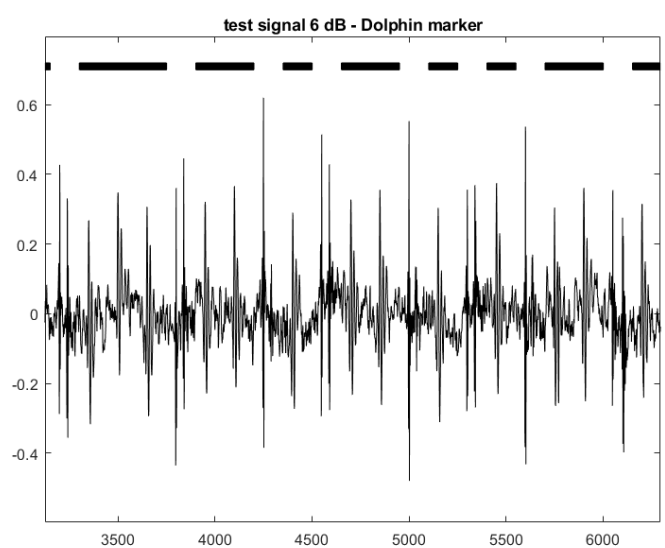

Fig. 4. Test Signal $($ SNRsea $=6 \mathrm{~dB}):$ zoom

\section{Correlation matrices}

Considering $N$ realizations $\boldsymbol{s}_{\boldsymbol{i}}$ (of length $n$ ) of a signal, the correlation matrix $\boldsymbol{C}$ is an $n \times n$-matrix of rank $N$ obtained by:

$$
\boldsymbol{C}=\sum_{i=1}^{N} \boldsymbol{s}_{\boldsymbol{i}} \boldsymbol{s}_{\boldsymbol{i}}^{\mathrm{T}}
$$

Unfortunately, we have $n=150$ but only 15 realizations available: in order not to take all the realizations for the estimation of the correlation matrix and keep some for the test signal, we choose $N=10$.

Using the SMF or CSMF theory, usually, correlation matrices are full-rank. Here, this is not the case and this is a serious difficulty: it is necessary to define a signal dolphin subspace which is of dimension $N=10$.

\section{Signal Processing and Results}

The usual processing consists in a mobile linear filtering of a piece of length n of the test signal $\boldsymbol{x}: \boldsymbol{X}^{\mathrm{T}} \boldsymbol{x}$ where $\boldsymbol{X}$ is a $n \times p$ matrix spanning the optimal $\mathrm{p}$-dimension subspace.

$\boldsymbol{X}^{\mathrm{T}} \boldsymbol{x}$ is a $p$-dimension vector and we calculate its square norm to create a signal to be thresholded and make a decision, called decision signal.

Given the above, a preprocessing stage is necessary: it consists in projecting $\boldsymbol{x}$ onto the signal dolphin subspace: the result is $\boldsymbol{x}^{\prime}$. In this subspace, we work with $\boldsymbol{A}^{\prime}, \boldsymbol{B}_{\mathbf{1}}^{\prime}$ and $\boldsymbol{B}_{\mathbf{2}}^{\prime}$ of dimension $N \times N$ and no more $\boldsymbol{A}, \boldsymbol{B}_{\mathbf{1}}$ and $\boldsymbol{B}_{\mathbf{2}}$ of dimension $n \times$ $n$.

Then we have $\boldsymbol{X}^{\prime}$ of dimension $N \times p$, and the decision signal is the square norm of $\boldsymbol{X}^{\prime \mathrm{T}} \boldsymbol{x}^{\prime}$.

The correlation matrices $\boldsymbol{A}^{\prime}, \boldsymbol{B}_{1}^{\prime}$ and $\boldsymbol{B}_{2}^{\prime}$ are full-rank but not normalized. Hence, the different criteria CSMF (arithmetic, geometric and harmonic) need the $\rho_{i}$ to be weighted according to the traces of the matrices.

Fig. 5 shows an example of the obtained decision signal for the multicriteria filter. Areas where dolphin signal is present are drawn with a dotted square function.

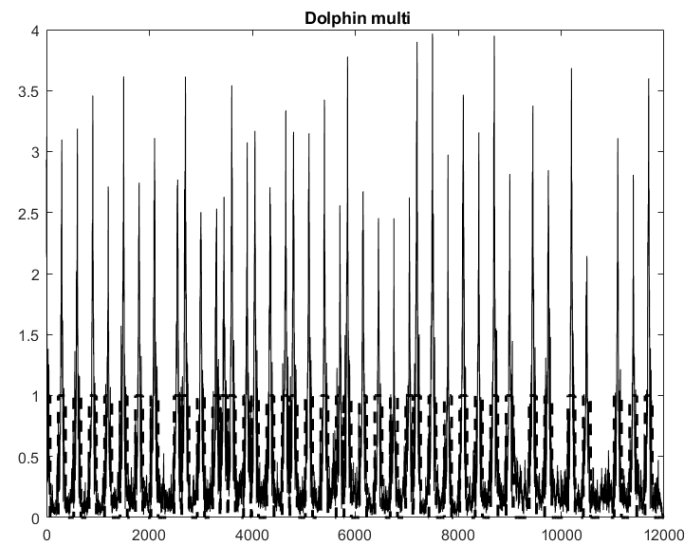

Fig. 5. Decision Signal $($ SNRsea $=6 \mathrm{~dB})$ 
The ROC curves are obtained with a varying threshold (from 0 to the max value). They are given on Fig. 6 and 7 .

On Fig. 6 are compared the results obtained with the three different CSMF presented above and the Multicriteria method (MCSMF).

The MCSMF is here slightly better than the others (equivalent to the geometric criterium).

On Fig. 7 are compared the results obtained with MCSMF and the SMF methods: the SMF where $\boldsymbol{B}=\boldsymbol{B}_{\mathbf{1}}$ is the sea noise correlation matrix (SMF Sea), and the SMF where $\boldsymbol{B}=\boldsymbol{B}_{\mathbf{1}}+$ $\boldsymbol{B}_{2}(\mathrm{SMF} \Sigma)$.

The MCSMF is here better than the others.

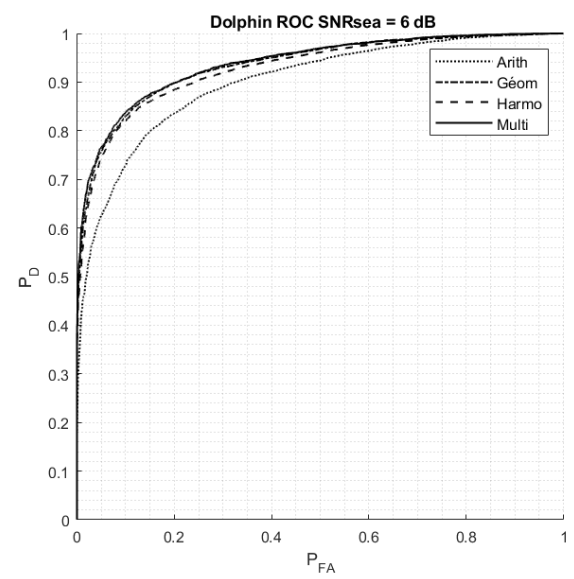

Fig. 6. ROC curve for CSMF methods (SNRsea $=6 \mathrm{~dB}$ )

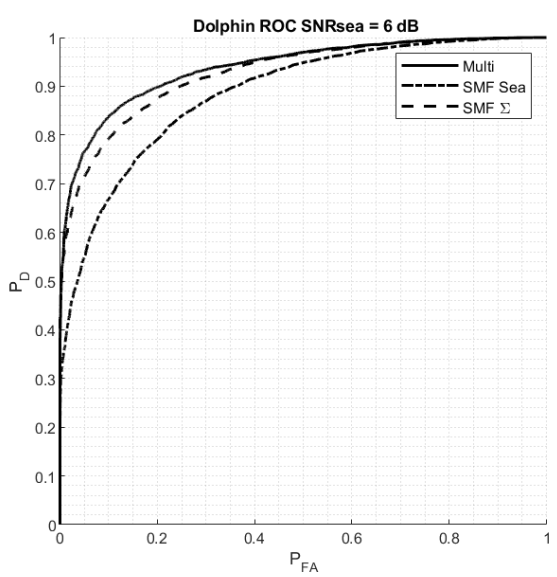

Fig. 7. ROC curve for MCSMF and SMF methods (SNRsea $=6 \mathrm{~dB}$ )
For a SNR Sea of $0 \mathrm{~dB}$, we can see the results on Fig. 8.

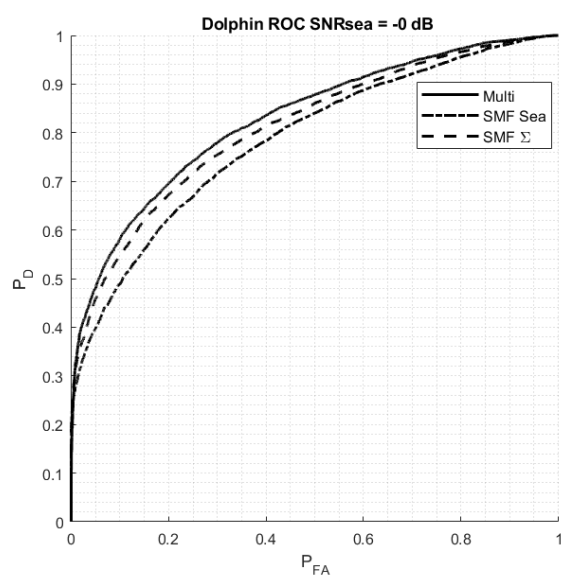

Fig. 8. ROC curve for MCSMF and SMF methods $($ SNRsea $=0 \mathrm{~dB})$

Note that for the detection of sperm whales, the MCSMF gives equivalent results than $\mathrm{SMF} \Sigma$ which is usually used.

The calculation of $P_{D}$ is performed as follows: we count the number of samples above the threshold in the detection areas and divide it by the total number of samples in the detection areas. Such an approach is very penalizing and underestimates the detection probability.

Here below is given an example of ROC curves for different values of $\mathrm{p}$ for the geometric CSMF. We can observe that the best result is for $p=5$.

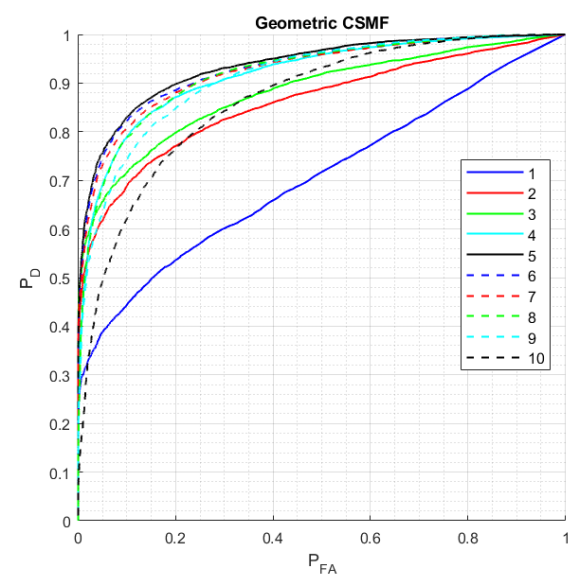

Fig. 9. ROC curves for geometric CSMF (SNRsea $=6 \mathrm{~dB})$ 


\section{CONCLUSION}

In this paper we attack the problem of the detection of a bioacoustic signal embedded in several noises such as sea noise and other bioacoustic signals (dolphins, sperm whales).

We propose an extension of the Constrained Stochastic Matched Filter (CSMF) based on the optimization of the Signal to Noise Ratio after linear filtering. The approach proposed is a multicriteria one, merging three different versions of the CSMF (arithmetic, geometric and harmonic criteria), and is named Multicriteria CSMF (MCSMF).

The MCSMF has been tested on real world bioacoustic signals and compared to the three versions of the CSMF and to the classical method Stochastic Matched Filter (SMF).

The results are provided on ROC curves. For dolphin detection, they are better for the MCSMF than for the other methods, or at least equal to the best among the three CSMF.

Different powers of the sea noise have been tested (SNR of $0 \mathrm{~dB}$ and $6 \mathrm{~dB})$ : obviously, the higher is this power, more difficult it is to discriminate the results of SMF and MCSMF.

For sperm whales detection, all the results are equivalents.

\section{REFERENCES}

[1] SABIOD Scaled Acoustic, BIODiversity platform, (http://sabiod.univtln.fr/data_samples.html)

[2] L. Bouffaut, R. Dreo, V. Labat, A.-O. Boudraa, et G. Barruol, Passive Stochastic Matched Filter for Antarctic blue whale call detection, The Journal of the Acoustical Society of America, vol 144, 2018.

[3] L. Bouffaut, R. Dreo, V. Labat, A.-O. Boudraa, et G. Barruol, Antarctic Blue Whale calls detection based on an improved version of the stochastic matched filter, 25 ${ }^{\text {th }}$ EUSIPCO, p. 2319-2323, 2017.

[4] P. Courmontagne et F. Chaillan, The Adaptive Stochastic Matched Filter for SAS Images denoising, dans OCEANS 2006, USA, p.1-6, 2006.

[5] B. Borloz et B. Xerri, Subspace SNR Maximization: The Constrained Stochastic Matched Filter, IEEE Transactions on Signal Processing, vol. 59, $\mathrm{n}^{\mathrm{o}}$ 4, p. 1346-1355, 2011.

[6] B. Borloz, Estimation, détection, classification par maximisation du rapport signal-à-bruit: le filtre adapté stochastique sous contrainte, thèse, Université de Toulon, 2005.

[7] M. Chagmani, Filtre adapté stochastique : poursuite de sous-espace et filtrage multicritère. Application à l'acoustique sous-marine, thèse, Université de Toulon, 2018. 